\title{
Outcomes of a brief coping skills group intervention for adults with severe postconcussion symptoms
}

\author{
Jordan I Ali ${ }^{1}$ (D), Patricia Mahoney², Derry Dance ${ }^{2,3}$ \& Noah D Silverberg*,2,3,4 \\ ${ }^{1}$ Department of Psychology, University of Victoria, Victoria, BC, V8W 2Y2, Canada \\ ${ }^{2}$ Acquired Brain Injury Program, GF Strong Rehabilitation Centre, Vancouver, BC, V5Z 2G9, Canada \\ ${ }^{3}$ Division of Physical Medicine \& Rehabilitation, University of British Columbia, Vancouver, BC, V5Z 2G9, Canada \\ ${ }^{4}$ Rehabilitation Research Program, Vancouver Coastal Health Research Institute, Vancouver, BC, V5Z 2G9, Canada \\ *Author for correspondence: Tel.: +1 604737 6249; Fax: +1 604737 6359; noah.silverberg@vch.ca
}

\begin{abstract}
Aim: The aim of this study was to evaluate a brief psychologically informed coping skills group intervention for adults with severe prolonged symptoms following mild traumatic brain injury (mTBI). Methodology \& results: Patients attended an education session about $\mathrm{mTBI} ; 22$ patients completed an additional coping skills group intervention, 16 declined/stopped the intervention early and 19 were not offered the intervention. At follow-up, patients who completed the intervention reported a similar degree of symptom improvement and disability as those who did not complete the intervention. The majority of patients who completed the intervention were satisfied with it and perceived it to be credible. Conclusion: The coping skills intervention was not associated with measurable clinical benefit. Recommendations for improving psychological interventions for mTBI are discussed.
\end{abstract}

First draft submitted: 9 August 2019; Accepted for publication: 23 September 2019; Published online: 21 November 2019

Keywords: concussion • mild TBI • postconcussion syndrome • rehabilitation • treatment

Mild traumatic brain injury (mTBI) symptoms are generally expected to remit within 3 months; however, a substantial minority of patients who sustain an mTBI will experience symptoms that persist over the longer term [1]. Psychosocial factors, such as anxiety and maladaptive illness beliefs, are increasingly understood to be important perpetuating factors of chronic symptoms [2,3]. In light of this, early psychologically informed intervention is considered a promising approach to prevent prolonged symptoms after mTBI. Early cognitive behavioral therapy (CBT) may be an effective complement to education and reassurance $[4,5,6]$, but the available evidence is limited and access to an individual therapist may be a barrier to implementation.

The current study describes a secondary analysis of a clinical quality improvement database compiled for the purposes of evaluating a brief CBT-informed coping skills group intervention for patients with severe prolonged symptoms after mTBI. Patients with severe, prolonged symptoms were targeted because they had the greatest need and potential to benefit from CBT and because CBT may be associated with a negligible group-level treatment effect when delivered to an unselected cohort [7]. The objectives of our secondary analysis were to provide preliminary evidence regarding the impact of the intervention on postconcussion symptom severity and self-reported disability. Further, we examined patient satisfaction with and perceived credibility of the coping skills intervention.

\section{Methods}

As a secondary analysis of a de-identified clinical database, the present study was exempt from University of British Columbia research ethics board approval but was conducted in accordance with the Declaration of Helsinki.

Eligible patients were adults (aged 19+ years) referred by a physician to a specialty outpatient program, the Early Response Concussion Service at the GF Strong Rehabilitation Centre (BC, Canada). Patients first attended a 2-hour group education session about recovery from $\mathrm{mTBI}$ and symptom self-management strategies, led by an occupational therapist. During the session, patients completed questionnaires regarding their postconcussion 


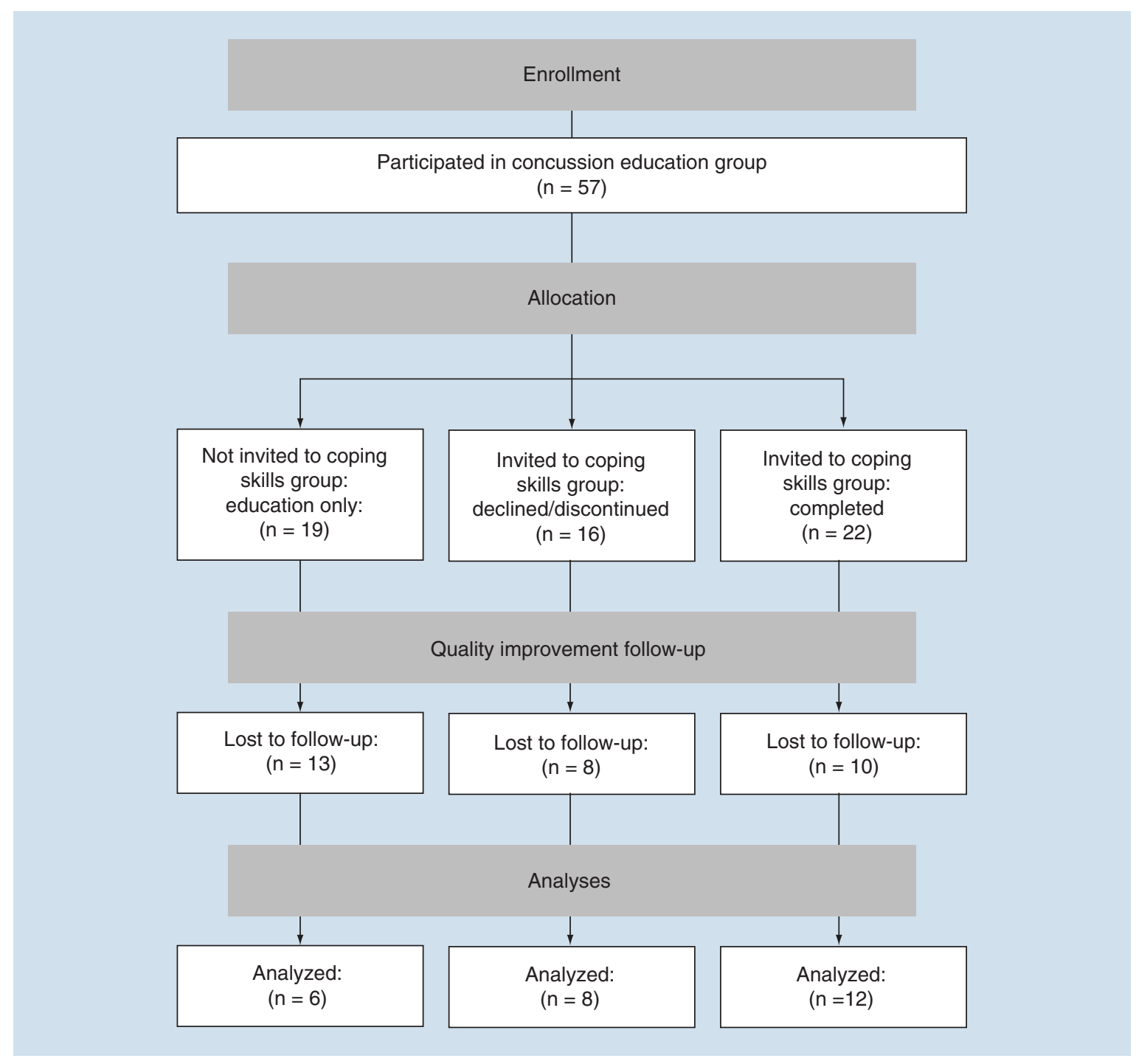

Figure 1. Patient flow.

symptoms (Rivermead Post Concussion Symptoms Questionnaire; RPQ [8]) and emotional health (Hospital Anxiety and Depression Scale; HADS [9]) until an interim analysis suggested that administering both measures was redundant for screening, as highly elevated RPQ scores $(>40)$ rarely occurred without correspondingly elevated HADS scores ( $>7$; frequency $=0 / 64,0 \%)$.

Education session attendees who were less than 3 months post-injury and met criteria for high symptom burden were considered eligible for additional intervention - the new coping skills group as described below. High symptom burden was defined as either RPQ $>40$ or both RPQ $>24$ and HADS anxiety or depression subscale $>7$ [9]. Note that RPQ $>24$ falls above the 90th percentile for clinical norms [10]. Some otherwise eligible patients were not offered the group because their program entry coincided with therapist unavailability (i.e., low staffing), creating a natural control group of patients who did not receive the intervention of interest. A clinician blinded to intervention condition attempted to contact patients for quality improvement purposes at a minimum of 3 months after their program exit. Available patients completed self-report measures by telephone. Figure 1 illustrates the flow of patients through the study.

\section{Coping skills group intervention}

Weekly 90-min sessions were co-delivered by an occupational therapist (P Mahoney) and a neuropsychologist (ND Silverberg) over 3 weeks, in cohorts of four to eight patients. Session 1 focused on developing a gradual return to 
activity plan and initiating its implementation. Session 2 oriented patients to the CBT model and how to apply cognitive reframing to common maladaptive illness beliefs after mTBI. In Session 3, participants were introduced to the overlap between symptoms of stress and symptoms of mTBI and were instructed on how to use relaxation strategies, including diaphragmatic breathing and progressive muscle relaxation. Patients completed homework assignments between sessions to assist with internalizing and generalizing these coping skills. A treatment manual with preprinted homework sheets was used to promote treatment fidelity. The full treatment manual is available from the authors upon request.

\section{Measures}

Rivermead Post Concussion Symptoms Questionnaire (RPQ) [8]. The RPQ is a reliable and frequently used measure of postconcussion symptom burden. Respondents rate their experience of 16 common postconcussion symptoms on a scale from 0 ('not experienced at all') to 4 ('a severe problem'). An RPQ score of 1 ('no more of a problem' following $\mathrm{mTBI}$ ) denotes that a given symptom was present prior to the mTBI and continues to be present at the same (pre-injury) severity level. To determine the severity of symptoms with onset or aggravation following mTBI specifically, a total symptom burden score was derived by summing all item scores $>1$.

World Health Organization Disability Assessment Schedule 2.0 12-item interview version (WHODAS) [11]. The WHODAS is a disease-nonspecific measure of six International Classification of Disability, Functioning and Health domains, including cognition, mobility, self-care, interpersonal functioning, life activities and participation. Respondents rate their level of disability in each domain on a scale from 0 ('None') to 4 ('Extreme/Cannot do'). The WHODAS is a reliable measure of disability after mTBI [11].

Patients who completed the coping skills group were also asked to rate their satisfaction with the coping skills intervention ('How satisfied were you with the coping skills group?') as well as 'How likely is it that (they) would recommend the coping skills group to a friend/family member in a similar situation?' on a scale from 1 ('not at all') to 5 ('extremely'). The latter question was adapted from a legacy instrument for measuring perceived credibility of psychotherapies [12].

\section{Analyses}

Demographic factors were compared across patients who completed the coping skills intervention, declined or discontinued the coping skills intervention (attended $<2$ of 3 sessions) or were not offered the coping skills intervention due to staffing limitations. Continuous variables were calculated using multivariate Analysis of Variance with group (three levels) as the independent variable and categorical variables were calculated using chi-squared tests. Demographic comparisons between patients available and unavailable for follow-up were conducted with independent samples t-tests. Pre-post symptom change (RPQ) and disability ratings (WHODAS) were calculated for patients who could be reached for follow-up. Total pre-post symptom change (RPQ) was calculated with repeated samples t-test. Group comparisons of disability (WHODAS) and rate of symptom change (RPQ) were conducted via univariate Analysis of Variance with group ( 3 levels) as the independent variable. A p-value $<0.05$ was considered statistically significant for all analyses.

\section{Results}

There were no significant differences between intervention conditions with regard to sex, age or time since injury. Patients who declined or discontinued the coping skills intervention had significantly less involvement in litigation/compensation claims versus those who completed the coping skills intervention or were not offered the coping skills intervention $\left(\chi^{2}[2, N=57]=8.56\right), \mathrm{p}=0.01$ (Table 1$)$. A total of 25 patients completed follow-up, one patient partially completed follow-up (excluding satisfaction and credibility items) and 31 patients either declined or were unavailable for follow-up after three attempts to contact them (Figure 1). There was no significant difference in intervention condition representation at follow-up. There were no significant differences in baseline characteristics between patients available and unavailable for follow-up, except that patients who completed followup endorsed significantly lower symptom burden $(\mathrm{RPQ})$ at intake than patients who did not $(\mathrm{t}[50]=2.25, \mathrm{p}=$ $0.03)$.

Symptom burden decreased over time $(\mathrm{t}[23]=5.2, \mathrm{p}<0.01)$, although remained elevated at follow-up $(\mathrm{RPQ}>24)$ across intervention conditions $(M=31.0, \mathrm{SD}=10.6)$. The degree of symptom reduction was comparable across intervention conditions $(F[2,21]=0.15, \mathrm{p}=0.87)$. Once differences in baseline symptom burden 


\begin{tabular}{|c|c|c|c|c|c|}
\hline Total $\mathbf{N}=57$ & $\begin{array}{l}\text { Coping skills: not } \\
\text { offered } \\
\text { Total } n=19 \\
\text { Follow-up } n=6\end{array}$ & $\begin{array}{l}\text { Coping skills: } \\
\text { declined/discontinued } \\
\text { Total } n=16 \\
\text { Follow-up } n=8\end{array}$ & $\begin{array}{l}\text { Coping skills: } \\
\text { completed } \\
\text { Total } n=22 \\
\text { Follow-up } n=12\end{array}$ & $\begin{array}{l}\text { Follow-up: } \\
\text { completed } \\
n=26\end{array}$ & $\begin{array}{l}\text { Follow-up: } \\
\text { declined/unavailable } \\
\mathrm{n}=31\end{array}$ \\
\hline Female $\mathrm{n}(\%)$ & $16(84.2)$ & $11(68.8)$ & $15(68.2)$ & $17(65.4)$ & $25(80.6)$ \\
\hline Age $M(S D)$ & $35.0(11.0)$ & $36.4(14.0)$ & $38.3(10.5)$ & $36.3(11.8)$ & $36.8(11.5)$ \\
\hline Personal injury litigation $\mathrm{n}(\%)$ & $10(52.6)$ & $1(6.3)^{\dagger}$ & $8(36.4)$ & $6(23.1)$ & $13(41.9)$ \\
\hline Motor vehicle accident $\mathrm{n}(\%)$ & $12(63.2)$ & $4(25.0)$ & $14(63.6)$ & $17(65.4)$ & $13(41.9)$ \\
\hline Weeks injury-clinic entry M (SD) & $6.2(3.0)$ & $5.1(3.3)$ & $6.3(3.5)$ & $6.4(3.3)$ & $5.6(3.2)$ \\
\hline Baseline RPQ M (SD) & $\begin{array}{l}42.5(10.8) \\
\mathrm{Cl}=38.4,46.6\end{array}$ & $\begin{array}{l}43.8(6.7) \\
\mathrm{Cl}=39.0,48.6\end{array}$ & $\begin{array}{l}45.0(8.1) \\
\mathrm{Cl}=40.9,49.0\end{array}$ & $\begin{array}{l}40.9(8.2)^{\dagger} \\
\mathrm{Cl}=37.5,44.4\end{array}$ & $\begin{array}{l}46.2(8.6)^{\dagger} \\
\mathrm{Cl}=43.0,49.4\end{array}$ \\
\hline Weeks injury-follow-up M (SD) & $35.0(3.6)$ & $29.2(5.2)$ & $35.7(4.8)$ & $33.8(5.4)$ & - \\
\hline Follow-up RPQ M (SD) & $\begin{array}{l}24.2(10.5) \\
\mathrm{Cl}=15.6,32.8\end{array}$ & $\begin{array}{l}31.5(11.0) \\
\mathrm{Cl}=24.0,39.0\end{array}$ & $\begin{array}{l}34.1(9.5) \\
\mathrm{Cl}=28.0,40.2\end{array}$ & $\begin{array}{l}31.0(10.6) \\
\mathrm{Cl}=26.7,35.27\end{array}$ & - \\
\hline RPQ change $M(S D)$ & $\begin{array}{l}9.3(6.5) \\
\mathrm{Cl}=0.9,17.8\end{array}$ & $\begin{array}{l}11.8(12.5) \\
\mathrm{Cl}=4.5,19.0\end{array}$ & $\begin{array}{l}9.5(9.2) \\
\mathrm{Cl}=3.0,16.0\end{array}$ & $\begin{array}{l}10.2(9.5)^{\dagger} \\
\mathrm{Cl}=6.2,14.2\end{array}$ & - \\
\hline WHODAS M (SD) & $\begin{array}{l}20.8(9.1) \\
\mathrm{Cl}=13.6,28.1\end{array}$ & $\begin{array}{l}27.3(9.2) \\
\mathrm{Cl}=21.0,33.5\end{array}$ & $\begin{array}{l}32.1(8.0) \\
\mathrm{Cl}=26.9,37.2\end{array}$ & $\begin{array}{l}28.0(9.4) \\
\mathrm{Cl}=24.2,31.8\end{array}$ & - \\
\hline
\end{tabular}

were accounted for, there was no significant difference in disability (WHODAS) between intervention conditions $(\mathrm{F}[2,20]=1.40, \mathrm{p}=0.27)$.

At follow-up, 73\% (8/11) of patients who completed the coping skills intervention reported that they were at least 'somewhat satisfied' with the group and $91 \%$ (10/11) would likely or very likely recommend it to a friend with mTBI.

\section{Discussion}

We conducted a secondary analysis of a clinical registry to evaluate the effectiveness of a brief coping skills group intervention for adults with high symptom burden following mTBI. We compared self-reported outcomes between participants who completed, declined/discontinued or were not offered the coping skills group because of staffing unavailability. Symptom burden decreased significantly over time; however, the rate of improvement was comparable across intervention conditions. Thus, our results do not support the effectiveness of this coping skills intervention beyond treatment as usual (e.g., psychoeducation). Nevertheless, patients reported moderate to high satisfaction with and perceived credibility of the intervention.

While it is possible that the coping skills intervention provided no clinical benefit beyond an in-person education session, there are several alternative reasons that may underlie our found null effects. Given that the small group differences favored those receiving no additional intervention, it is unlikely that one of these reasons was insufficient statistical power. Rather, one possible reason is that the intervention was not sufficiently potent. It was designed to be very brief for feasibility purposes, but this may have come at a cost to effectiveness. It is possible that a larger or more concentrated 'dose' of therapy may be required, especially for highly symptomatic patients at risk for poor outcomes. Indeed, a three-session group is a very low dose for typical CBT trials [13,14]. Expanding on the content over a greater number of sessions or supplementing or replacing the group sessions with individual support may increase intervention effectiveness. Our use of a group format may help reconcile differences with prior studies that found CBT to be effective following $\mathrm{mTBI}$, using individual therapy $[5,15]$. Potential benefits of group therapy (e.g., symptom normalization, enhanced motivation, modeling adaptive coping) [16] may be outweighed by disadvantages (e.g., symptom contagion or other iatrogenic effects) in the context of mTBI.

Additionally, the outcome measures used in this study may have been too indirect and, therefore, insensitive to potential intervention effects. Although the direct intervention targets were coping, self-efficacy and modified illness perceptions, the outcome measures narrowly assessed the number and intensity of symptoms. Another potential reason for the lack of apparent intervention effect is the patient selection method. Patients were invited to the group on the basis of being highly symptomatic; however, it may be more productive to offer CBT to patients who demonstrate maladaptive coping instead (i.e., who display the specific psychological risk factors that CBT is 
designed to modify). Finally, patients who declined or were not offered the coping skills intervention may have been more likely to receive additional private treatment.

\section{Study limitations}

Several study limitations warrant mentioning. First, participants were not randomly assigned to treatment conditions. Some were not offered the intervention of interest based on inadequate staffing availability at the time they presented to clinic, creating a comparison (no additional intervention control) group. The decision to offer versus not offer the coping skills group was independent of patient characteristics and so can be considered pseudo-random. Nevertheless, the intervention groups appeared generally comparable on measured demographic and injury characteristics, as well as baseline symptom severity. Another major limitation was high (55\%) loss to follow-up, particularly of those with highest initial symptom burden. This degree of missing follow-up data and the likelihood of nonrandom mechanisms contributing to missingness may be an important source of bias in this study, further tempering conclusions about treatment (in)effectiveness. Additionally, we relied on the referring physician to diagnose mTBI. We analyzed data from a clinical program that did not impose particular diagnostic criteria on referrers or provide routine diagnostic verification. Finally, because the intervention was delivered in a clinical context, the sessions were not audio- or video-recorded, and so assessment of therapist fidelity to the treatment manual was not possible.

Future research may benefit from true randomization and more purposeful pre-post assessment design using measures specifically matched to intervention content or proposed mechanisms of therapeutic action. Last, the exploration of longer group formats or supplementation with individual therapy may prove fruitful.

\section{Conclusion}

In this nonrandomized trial, treatment-seeking patients with high symptom burden after mTBI who completed a brief CBT-based coping skills group intervention perceived the group to be satisfactory and credible, but their symptom recovery trajectory and disability outcomes were comparable to patients who did not complete the group. More intensive intervention may be required for this patient subpopulation.

\section{Future perspective}

Psychological factors are increasingly recognized as potent modifiers of recovery from mTBI. Over the next decade, we expect that evidence-based theoretical models will advance to specify which psychological factors are most important and how they (mechanistically) lead to symptom chronicity. We will be able to apply principles of precision medicine to better identify patients with particular psychological risk factors for poor mTBI outcome and target therapy to those risk factors. The optimal timing, dose and format of psychologically informed treatment will also be better established.

\section{Summary points}

\section{Rationale}

- Psychosocial factors are important perpetuating factors of prolonged symptoms following mild traumatic brain injury (mTBI).

- Early psychotherapeutic intervention may be beneficial to those with mTBI, particularly those with severe symptoms.

Intervention

- A three-session coping skills group intervention was designed to help patients develop a graduated return to activity plan and learn how to use cognitive reframing and relaxation techniques.

Results

- At follow-up, patients who completed the coping skills intervention reported no more symptom improvement than patients who had received treatment as usual.

- The majority of patients who completed the coping skills intervention reported that they were at least 'somewhat satisfied' with the group and would likely or very likely recommend it to a friend with mTBI.

Recommendations

- More intensive intervention (e.g., more sessions) may be necessary for this patient subpopulation.

- Future studies would benefit from true randomization and selection of outcome measures matched to the specific content of the intervention. 
Financial \& competing interests disclosure

ND Silverberg receives personal support from the Michael Smith Foundation (Health Professional Investigator Award), as well as institutional support via the Canadian Institutes of Health Research, VGH and UBC Hospital Foundation. Jl Ali receives financial support from the Alzheimer Society of Canada (Dr \& Mrs Albert Spatz Award, 18-29). The authors have no other relevant affiliations or financial involvement with any organization or entity with a financial interest in or financial conflict with the subject matter or materials discussed in the manuscript apart from those disclosed.

No writing assistance was utilized in the production of this manuscript.

Ethical conduct of research

The authors state that they have obtained appropriate institutional review board approval or have followed the principles outlined in the Declaration of Helsinki for all human or animal experimental investigations. In addition, for investigations involving human subjects, informed consent has been obtained from the participants involved.

Open access

This work is licensed under the Creative Commons Attribution 4.0 License. To view a copy of this license, visit http://creativecomm ons.org/licenses/by/4.0/

\section{References}

Papers of special note have been highlighted as: $\bullet$ of interest

1. Maas AIR, Menon DK, Adelson PD et al. Traumatic brain injury: integrated approaches to improve prevention, clinical care and research. Lancet Neurol. 16(12), 987-1048 (2017).

- Provides a comprehensive review of the clinical definitions, epidemiology and impacts of traumatic brain injury (TBI) and mild traumatic brain injury (mTBI).

2. Broshek DK, De Marco AP, Freeman JR. A review of post-concussion syndrome and psychological factors associated with concussion. Brain Inj. 29(2), 228-237 (2015).

- Presents several psychological and neurobiological models relevant to common symptoms following $\mathrm{mTBI} /$ concussion and their longer-term perpetuation.

3. Silverberg ND, Gardner AJ, Brubacher JR, Panenka WJ, Li JJ, Iverson GL. Systematic review of multivariable prognostic models for mild traumatic brain injury. J. Neurotrauma 32(8), 517-526 (2015).

- Identifies pre-injury mental health, early post-injury neuropsychological functioning and early post-injury anxiety as the most robust predictors of $\mathrm{mTBI}$ outcome.

4. Mittenberg W, Tremont G, Zielinski RE, Fichera S, Rayls KR. Cognitive-behavioral prevention of postconcussion syndrome. Arch. Clin. Neuropsychol. 11(2), 139-145 (1996).

- Patients with mTBI received a tailored cognitive behavioral therapy-based intervention. The treatment group demonstrated unique improvements in headache, fatigue and cognitive functioning versus controls.

5. Silverberg ND, Hallam BJ, Rose A et al. Cognitive-behavioral prevention of postconcussion syndrome in at-risk patients: a pilot randomized controlled trial. J. Head Trauma Rehabil. 28(4), 313-322 (2013).

- Patients with $\mathbf{m T B I}$ received individual cognitive behavioral therapy tailored for $\mathrm{mTBI}$ symptoms. The treatment group demonstrated improved symptom burden, mood and daily function compared with controls.

6. Snell DL, Surgenor LJ, Hay-smith EJC, Siegert RJ. A systematic review of psychological treatments for mild traumatic brain injury: an update on the evidence. J. Clin. Exp. Neuropsychol. 31(1), 20-38 (2008).

- Reviews psychological interventions for mTBI. They concluded that the literature was too heterogeneous to draw firm conclusions regarding the efficacy of specific interventions.

7. Scheenen ME, Visser-Keizer AC, de Koning ME et al. Cognitive behavioral intervention compared with telephone counseling early after mild traumatic brain injury: a randomized trial. J. Neurotrauma 34(19), 2713-2720 (2017).

8. King NS, Crawford S, Wenden FJ, Moss NE, Wade DT. The Rivermead Post Concussion Symptoms Questionnaire: a measure of symptoms commonly experienced after head injury and its reliability. J. Neurol. 242(9), 587-592 (1995).

9. Zigmond AS, Snaith RP. The hospital anxiety and depression scale. Acta Psychiatr. Scand. 67(6), 361-370 (1983).

10. Potter S, Leigh E, Wade D, Fleminger S. The Rivermead Post Concussion Symptoms Questionnaire: a confirmatory factor analysis. J. Neurol. 253(12), 1603-1614 (2006).

11. Snell DL, Iverson GL, Panenka WJ, Silverberg ND. Preliminary validation of the World Health Organization Disability Assessment Schedule 2.0 for mild traumatic brain injury. J. Neurotrauma 34(23), 3256-3261 (2017).

12. Borkovec TD, Nau SD. Credibility of analogue therapy rationales. J. Behav. Ther. Exp. Psychiatry 3(4), 257-260 (1972). 
13. Okumura Y, Ichikura K. Efficacy and acceptability of group cognitive behavioral therapy for depression: a systematic review and meta-analysis. J. Affect. Disord. 164, 155-164 (2014).

14. Bernardy K, Füber N, Köllner V, Häuser W. Efficacy of cognitive-behavioral therapies in fibromyalgia syndrome - a systematic review and metaanalysis of randomized controlled trials. J. Rheumatol. 37(10), 1991-2005 (2010).

15. Tomfohr-Madsen L, Madsen JW, Bonneville D et al. A pilot randomized controlled trial of cognitive behavioral therapy for insomnia in adolescents with persistent postconcussion symptoms. J. Head Trauma Rehabil. doi:10.1097/HTR.0000000000000504 (2019) (Epub ahead of print).

16. Thastum MM, Rask CU, Naess-Schmidt ET et al. Design of an early intervention for persistent post-concussion symptoms in adolescents and young adults: a feasibility study. NeuroRehabilitation 43(2), 155-167 (2018). 